\title{
The Relationship Between Musculoskeletal Pains, Serum Estradiol Level and Climacteric Symptoms in Postmenopausal Women
}

Neda Mohamadzade

Tarbiat Modares University Faculty of Medical Sciences

Shahideh Jahanian Sadatmahalleh

Tarbiat Modares University Faculty of Medical Sciences

Saeideh Ziaei ( $\boldsymbol{D}$ Ziaei_Sa@modares.ac.ir)

Tarbiat Modares University Faculty of Medical Sciences https://orcid.org/0000-0002-6297-9926

Narges Zaeemzadeh

Tarbiat Modares University Faculty of Medical Sciences

Anoshirvan Kazemnejad

Tarbiat Modares University Faculty of Medical Sciences

\section{Research}

Keywords: Climacteric Symptoms, Estradiol, Musculoskeletal Pains

Posted Date: June 17th, 2020

DOI: https://doi.org/10.21203/rs.3.rs-34791/v1

License: (c) (i) This work is licensed under a Creative Commons Attribution 4.0 International License. Read Full License 


\section{Abstract}

Background: Due to the decrease of estrogen levels in postmenopausal females, menopause can be associated with musculoskeletal pains. This study was designed to assess the possible association between musculoskeletal pains, serum estradiol level, and climacteric symptoms in postmenopausal women.

Methods: This cross-sectional study was conducted on 307 postmenopausal females selected by convenience sampling method. Data collection procedure lasted from October 2016 to September 2018. The required data were collected using the Menopause Rating Scale (MRS), Örebro Musculoskeletal Pain Questionnaire (ÖMPQ), and a questionnaire containing personal data. The blood sample was taken to measure the estradiol level.

Results: There was a direct relationship between musculoskeletal pains and menopause symptoms $(p<0.001)$, age $(p=0.03)$, parity $(p=0.01)$, and BMI $(p=0.03)$ and an indirect association between musculoskeletal pain and marriage age $(p=0.009)$, age of first pregnancy $(0.017)$, estradiol level $(p<0.001)$ and education level $(p=0.002)$. The regression analysis results showed that menopause symptoms were the strongest predictor of musculoskeletal pains among all the variables.

Conclusions: The findings of the present study showed that although various parameters are associated with musculoskeletal pains, climacteric symptoms are the most important predictive parameters of musculoskeletal pains.

\section{Introduction}

Musculoskeletal disorders are known as the most important reasons of disability worldwide (1). Women report more regions of pain than men (2) may be under the influence of hormonal changes (3). Complaining of musculoskeletal pains is more prevalent than complaints about hot flashes among the women of menopausal age (4). Musculoskeletal pains can be associated with menopausal variables, especially vasomotor symptoms, which can show the role of hormonal changes in the pathogenesis of musculoskeletal pains (5). After the menopause, the ovaries stop producing a considerable amount of estrogen (6). Differences in serum estradiol levels will induce changes in the activity of the central nervous system, which will explain different symptoms in menopausal women (7). Although estrogens do not have a direct effect on articular muscle tissue, they can affect producing cytokines and can lead to an increase in nitric oxide production by endothelial cells. Cells that carry estrogen receptors release messenger RNA (mRNA) for enkephalins, which inhibit the nerve cells involved in pain perception (8). Musculoskeletal pains are mostly seen in menopausal women (9), and to a great extent, affect their personal and social life, job opportunities, and quality of life (10). 
Women experience new hormonal changes during the menopausal period, which can cause numerous problems if not paid due attention before this period. There are conflicting results related to the effect of estrogen or hormone therapy on musculoskeletal pains $(11,12)$. In addition, to our knowledge, there are no studies about the relationship between musculoskeletal pains, serum estradiol level, and climacteric symptoms in postmenopausal women. Therefore, this study was designed to assess associations between musculoskeletal pains, serum estradiol levels, and climacteric symptoms in postmenopausal women.

\section{Materials And Methods}

In a pilot study, a sample size calculation was performed using the correlation between musculoskeletal pains and serum estradiol levels. According to preliminary results with confidence interval $=95 \%$ and study power $=80 \%$, the appropriate sample size was calculated to be about 300 , which was increased to 307 women considering the probability of sample dropping. In this cross-sectional study, 307 postmenopausal women with musculoskeletal pains, in Tehran, Iran were enrolled. Data collection procedure lasted from October 2016 to September 2018, and a convenience sampling method was used for the study. The eligibility criteria for this study included: 1) age 45-65 years, 2) postmenopausal women (at least 12 months of amenorrhea), 3) having an intact uterus and ovaries, 4) no hormone therapy in the past 6 months, 5) no use of NSAIDs during the last month, 6) no history of chronic disease (cardiovascular disease, thyroid disease, cancer, osteoporosis, ...), 7) no history of alcohol consumption and cigarette smoking, and 8) without any misadventure during the past 6 months.

This study was conducted with the approval of the Ethics Committee of Tarbiat Modares University of Medical Sciences (IRB \# 525500). All the women were informed about the project and fulfilled the written consent before participating in the study. Then demographics, Örebro musculoskeletal pain, and Menopause Rating Scale (MRS) questionnaires were filled out. Estradiol levels were detected via electrochemiluminescence using a Cobas E411 analyzer (Roche Instr kit, Germany). Body mass index (BMI) was calculated as weight in kilograms divided by height in meters squared. Women were divided into three groups based on the frequency of their physical activity (at least, 20 min per week): no physical activity, 1-2 times of physical activity per week, and 3 or more times of physical activity per week (13).

Questionnaires

\section{Menopause Rating Scale (MRS)}

MRS is a standardized tool for assessing climacteric symptoms. It evaluates the presence and severity of symptoms/complaints related to menopause. The questionnaire consists of 11 items divided into three dimensions: a) Somato-Vegetative domain: hot flushes, cardiac complaints, sleeping disorders, and muscle and joint complaints; b) Psychological domain: depressed mood, irritability, anxiety, and mental and physical fatigue; and c) Urogenital domain: sexual problems, bladder complaints, and vaginal dryness. Each of these items can get the score of 0 - 4 as follows: $0=$ none, $1=$ mild, $2=$ moderate, $3=$ severe and $4=$ very severe. The total score of the MRS is calculated as the sum of the dimension scores 
and can range from 0 to 44 . Moreover, the participants were classified based on "severity of complaints" into four categories: no/little symptoms (0-4), mild (5-8), moderate (9-16), and severe complaints (17+) (14).

\section{Örebro Musculoskeletal Pain Questionnaire (ÖMPQ)}

ÖMPQ is a clinical instrument to identify patients at risk for chronic or prolonged musculoskeletal disorders. This questionnaire has 25 questions consisting of 21 items rated on a scale of 0 to 10 . Total scores are computed from 21 items and the maximum total score is 210 (15). In the present study, the participants were classified into three sub-scales (16): women with low risk for long-term disability (ÖMPQ <90), women with moderate risk for long-term disability (ÖMPQ = 90-105) and women with high risk for long-term disability (ÖMPQ > 105).

\section{Statistical Analysis}

The analysis of the collected data was carried out by the SPSS software (ver. 20). The normal distribution of the quantitative variables was assessed by Kolmogorov-Smirnov's test. For non-normal distributions, a non-parametric Spearman correlation test was used. Independent t-student test was used in order to determine the score difference of musculoskeletal pains in the two physical activity groups (with or without physical activity). Chi-square test was used to evaluate the qualitative variables. Also, stepwise multiple linear regression analysis was applied to identify the predictors of musculoskeletal pains. Significance levels were assumed for $p<0.05$.

\section{Results}

In the present study, 307 post-menopausal women who met the inclusion criteria were examined. Overall, mean age, age of menarche, age at marriage, age at first pregnancy, parity and age at menopause were $56.47 \pm 4.6,13.50 \pm 1.54,18.89 \pm 5.06,20.43 \pm 5.07,4.02 \pm 2.06$ and $49.19 \pm 3.83$, respectively. The mean score of the MRS and ÖMPQ was $12.81 \pm 4.65$ and $87.11 \pm 21.14$, respectively. As shown in Table 1, women with moderate menopausal symptoms $(60.3 \%)$ and low risk for long-term disability due to musculoskeletal pain $(51.5 \%)$ have the most frequency. 
Table 1

Frequency of demographic characteristics, reproductive information, MRS and ÖMPQ scores

\begin{tabular}{|ll|}
\hline Variable & $\mathbf{N}(\%)$ \\
\hline BMI (kg/m²) & $2(0.7)$ \\
Under weight & $71(23.1)$ \\
Normal weight & $140(45.6)$ \\
Over weight & $94(30.6)$ \\
Obese & \\
\hline Marriage status & $250(81.4)$ \\
Married & $57(18.5)$ \\
Divorced & \\
\hline Education status & $91(29.6)$ \\
Primary school & $153(49.8)$ \\
High school & $63(20.5)$ \\
University or above & \\
\hline Economic status & $85(27.7)$ \\
Low & $145(47.2)$ \\
Moderate & $77(25.1)$ \\
High & \\
\hline Physical activity & $200(65.1)$ \\
No & $65(21.2)$ \\
1-2 times a week & $42(13.7)$ \\
\hline or more times a week & \\
\hline ÖMPQ & $158(51.5)$ \\
< 90 & $88(28.7)$ \\
$90-105$ & $61(19.9)$ \\
\hline 105 & \\
\hline
\end{tabular}




\begin{tabular}{|ll|}
\hline Variable & $\mathbf{N}(\%)$ \\
\hline MRS & $3(1.0)$ \\
No, little (0-4) & $55(17.9)$ \\
Mild (5-8) & $185(60.3)$ \\
Moderate (9-16) & $64(20.8)$ \\
Severe (17+) & \\
\hline
\end{tabular}

In this study, the majority of the women were overweight (45.6\%) based on BMI, high school (32.2\% (, had moderate socio-economic status (47.2\%), and did no exercise (65.1\%). Table 2 shows a direct relationship between musculoskeletal pains and menopause symptoms $(p<0.001)$, age $(p=0.03)$, parity $(p=0.01)$, and BMI ( $p=0.03)$, and an indirect relationship between musculoskeletal pain and age at marriage $(p=$ $0.009)$, age at first pregnancy $(0.017)$, estradiol levels $(p<0.001)$ and education level $(p=0.002)$. In order to analyze the score difference of musculoskeletal pains in the two physical activity groups, independent t-student test was used. The mean \pm SD for the women with physical activity was $83.28 \pm 23.26$, and for those without physical activity, it was $89.16 \pm 19.66$; a significant difference was observed between the two groups $(p=0.02)$. There was a significant positive association between musculoskeletal pains and climacteric symptoms $(p<0.001)$. According to Table 3 , the Chi-square test results showed a significant difference between the severity of climacteric symptoms and the three categories of musculoskeletal pains $(p=0.001)$. In women with mild or without menopause symptoms there is not high risk for longterm disability, whereas, in those with severe menopause symptoms, this rate is $59.4 \%$. Stepwise linear multiple regression analysis was used to test the influence of the independent variables (age, age at first pregnancy, gravidity, BMI, education, level of serum estradiol, physical activity, age at marriage, and total score of the MRS) on musculoskeletal pains. The results revealed that menopause symptoms were a strong predictor of musculoskeletal pains among all the variables. 
Table 2

Correlation of demographic characteristics, reproductive information, climacteric symptoms and estradiol level with musculoskeletal pains

\begin{tabular}{|lll|}
\hline Variable & $\mathbf{r}$ & p-value $^{*}$ \\
\hline Age (years) & 0.12 & 0.03 \\
\hline Menarche age (years) & -0.01 & 0.82 \\
\hline marriage age (years) & -0.15 & 0.009 \\
\hline Age at first pregnancy (years) & -0.13 & 0.017 \\
\hline Parity & 0.13 & 0.015 \\
\hline Menopausal age (years) & 0.09 & 0.08 \\
\hline BMl (kg/m ${ }^{2}$ ) & 0.12 & 0.03 \\
\hline Estradiol (pg/ml) & -0.22 & $<0.001$ \\
\hline Educational status & -0.17 & 0.002 \\
\hline Economic status & -0.073 & 0.19 \\
\hline Climacteric symptoms & 0.627 & $<0.001$ \\
\hline * P-values refer to the non-parametric Spearman's correlation test. Statistical significance was set at \\
\hline p< 0.05
\end{tabular}


Table 3

Comparison of the severity frequency of climacteric symptoms at different levels of musculoskeletal pains

\begin{tabular}{|c|c|c|c|}
\hline \multirow[t]{3}{*}{ MRS } & \multicolumn{3}{|l|}{ ÖMPQ } \\
\hline & $\begin{array}{l}\text { Low risk for prolonged } \\
\text { disability }\end{array}$ & $\begin{array}{l}\text { Moderate risk for prolonged } \\
\text { disability }\end{array}$ & $\begin{array}{l}\text { High risk for prolonged } \\
\text { disability }\end{array}$ \\
\hline & $N(\%)$ & $\mathbf{N}(\%)$ & $\mathbf{N}(\%)$ \\
\hline No, little & $3(100 \%)$ & 0 & 0 \\
\hline Mild & $46(83.6 \%)$ & $9(16.4 \%)$ & 0 \\
\hline Moderate & $99(53.5 \%)$ & $63(34.1 \%)$ & $23(12.4 \%)$ \\
\hline Severe & $10(15.6 \%)$ & $16(25 \%)$ & $38(59.4 \%)$ \\
\hline P-value * & $P<0.001$ & & \\
\hline \multicolumn{4}{|c|}{ MRS; Menopause Rating Scale } \\
\hline \multicolumn{4}{|c|}{ ÖMPQ; Örebro Musculoskeletal Pain Questionnaire } \\
\hline
\end{tabular}

Table 4

Risk factors related to musculoskeletal pains: Stepwise liner regression analysis

\begin{tabular}{|llllll|} 
Variable & B & SE & Beta & P-value * & R $^{2}$ \\
\cline { 1 - 1 } & 2.818 & 0.208 & 0.621 & $\mathrm{p}<0.001$ & 0.384 \\
Constant & 51.182 & 2.846 & & & \\
\hline
\end{tabular}

MRS; Menopause Rating Scale

Dependent variable: Örebro Musculoskeletal Pain Questionnaire

Independent variables: age, first pregnancy age, gravidity, BMI, education, level of serum estradiol, physical activity, marriage age, and MRS.

* Statistical significance was set at $p<0.05$.

\section{Discussion}

The present study results indicated that there is a direct meaningful relationship between menopause symptoms and musculoskeletal pains in a way that women with severe climacteric symptoms reported higher rates of musculoskeletal pains. The women with mild or without menopause symptoms were not at high risk for long-term disability due to musculoskeletal pains, whereas in the women with severe menopause symptoms, this rate was $59.4 \%$. In a cross-sectional study by Juan E. Blumel, the prevalence 
of muscle and joint aches was $63 \%$, and $15.6 \%$ of which has severe and very severe musculoskeletal pain scores. In this large sample of mid-aged women, the prevalence of musculoskeletal pains was high, which was significantly related to menopausal variables; especially vasomotor symptoms. Also, only $8.2 \%$ of the women with no vasomotor symptoms suffered from severe/very severe muscle and joint aches, whereas in the women with very severe vasomotor symptoms, the prevalence of muscle and joint aches increased up to $60.2 \%$ (5). Korean research reported that climacteric symptoms and leisure time inactivity were statistically effective factors for low back pain in postmenopausal women. Women with backache expressed more severe climacteric symptoms than those without backache (4). Both of the above studies are relatively in accordance with our study. Similarly, in the study by Mitchell et al., a significant increase in backache was observed during the early postmenopause and women with vasomotor symptoms, depressed mood, anxiety, difficulty in concentrating and insomnia reported more back pain (12).

The results of these studies and our study highlight the fact that climacteric symptoms are directly associated with increases in musculoskeletal pains, which probably indicate the role of hormonal changes in this period. In our study, another factor related to musculoskeletal pains was serum estradiol level such that the declining serum estradiol level in the postmenopausal females promoted the development of musculoskeletal pains. By contrast, in Akkus's et al.'s study, no significant difference was seen in the measured values for estradiol between fibromyalgia patients and healthy females. Since the subjects' population consisted of both pre- and post-menopausal women, no significant difference between the groups appears to be influenced by hormonal fluctuations in pre-menopausal women (17). Sambrook et al. measured estrogen concentrations in 49 postmenopausal women with rheumatoid arthritis and 49 women without rheumatoid arthritis. In this study, compared with the control group, estrogen levels declined in the females with rheumatoid arthritis significantly (18).

In line with our findings, in Nikolv et al.'s study, there was a significant difference in the severity of back pain between women with low and women with normal levels of estrogen. In these women, plasma estrogen levels, as a hormonal and reproductive factor, had a significant negative relationship with low back pain (19).

Similarly, in Sowers et al.'s study, there was a significant association between low estradiol level, 2hydroxy estrone, and the development of knee osteoarthritis (20).

Estrogen is an important factor in maintaining the integration of the musculoskeletal system (21). The inability to produce estrogen in the menopausal status is associated with decreasing the skeletal muscle mass, muscle performance, and functional capacity (22). At present, there is growing evidence on the role of estrogen in increasing the activity of joint tissues via complex molecular pathways. Estrogen therapy plays an influential role in maintaining and restoring joint tissues in osteoarthritis (23). Stening et al conducted a clinical trial study involving 29 postmenopausal women. In this study, after six weeks of treatment with transdermal estrogen, no relief of pain was observed (24), which could be expectable because of the small sample size and short duration of estrogen therapy. Indeed, the effect of hormone 
therapy on pain possibly depends on the dose or duration of hormone therapy and also its different effects on the target organs such as the joints, spine, etc (25). Contrary to this, in a clinical trial study, 16,608 postmenopausal women were classified into conjugated estrogen plus medroxyprogesterone acetate or placebo. It was seen that the hormone therapy group reported relief of joint pain and joint stiffness (26).

As estrogen level decreases during menopause, this can induce musculoskeletal pains and joint stiffness; as a result, estrogen replacement therapy may decrease the risk of musculoskeletal pains.

Since menopause is not essentially a risk factor for musculoskeletal pains, it is important to identify other factors for this among menopausal women. In the present study, in addition to climacteric symptoms and serum estradiol level, older age, high parity, and high BMI were related to the higher risk of musculoskeletal pains. Furthermore, with the increase of marriage age, young maternal age, and physical activity, musculoskeletal pains decreased. In Wijnhoven et al.'s cross-sectional study, young maternal age and estrogen therapy during menopause were related to low back pain. The findings suggest that hormonal and reproductive factors are related to musculoskeletal pains (27). In explaining this finding, it seems that women have more severe menopausal symptoms, seek different treatments such as hormone therapy.

Meanwhile, in Sievert et al.'s study, menopausal women with back pain were more likely to be older, have less educational level, and have higher BMl, whereas women with joint pain were more likely to be postmenopause, with less educational level, more children and higher BMI (28). In Gao HL et al.'s study, menopause was regarded as a period for the increase of musculoskeletal symptoms. In addition, higher $\mathrm{BMI}$ and age were associated with increased prevalence of knee pain and joint stiffness (29).

The consistent message in the literature is that exercise is a safe and powerful tool to prevent and treat many medical, psychological, and musculoskeletal conditions in females at midlife and beyond (30).

Conversely, Mitchell et al. observed that more physical activity was associated with joint pain among menopausal women (12). The feasible explanation of this contradictory result may be due to the lack of international questionnaire to assess musculoskeletal pains in inactive women.

Females with low back pain avoid doing physical activity due to fear of pain; this can lead to a faulty cycle. Sedentary lifestyles promote muscle weakness and backache (4).

In our study, the results derived from stepwise linear multiple regression analysis showed menopause symptoms are a strong predictor of musculoskeletal pains among all the variables. As stated before, a few studies have been conducted on the relationship between climacteric symptoms and musculoskeletal pains but none of them has investigated the impact of climacteric symptoms in comparison with other factors such as serum estradiol level as well as reproductive and demographic data, and this may be seen as a potential strength of our study. However, some limitations were identified in this study. This work was a cross-sectional study, so we could not recognize the causality; rather only 
the relationship between climacteric symptoms, serum estradiol level, and musculoskeletal pains was targeted.

\section{Conclusion}

The findings of the present study showed that although various factors are associated with musculoskeletal pains, climacteric symptoms are the most important predictive parameters of musculoskeletal pains. Therefore, the rate of musculoskeletal pains may be reduced with the improvement and treatment of climacteric symptoms. Consequently, conducting clinical trials in order to treat climacteric symptoms for the evaluation of musculoskeletal pains is recommended.

\section{Declarations}

\section{Ethics approval and consent to participate}

After observing ethical and research principles, including receiving a code of ethics from the Research Ethics Committee of Tarbiat Modarres University (IRB \# 525500), submitting an introduction to sex clinics and explaining the nature and objectives of the study, the questionnaires were distributed and completed. After explaining the study's aims, written consent and verbal assent were obtained from women, and women were informed that their participation were voluntary, confidential, and anonymous, and were apprised of their right to withdraw from the research at any time. Participants were assured that the information in the questionnaire would be used confidentially.

\section{Consent for publication}

Not applicable.

\section{Availability of data and materials}

The data sets used and analyzed during the current study are available from the corresponding author on reasonable request.

\section{Competing interests}

The authors declare no conflict of interest.

\section{Funding}

None.

\section{Authors' contributions}

S.Z, Sh.JS designed and developed the project including the concepts of investigation; N.M and N.Z was involved in the acquisition of data; A.K, S.Z, Sh.JS analyzed and interpreted the data; S.Z, Sh.JS was 
involved in drafting of the manuscript; S.Z, N.M, N.Z performed critical revision of the manuscript for important intellectual content; S.Z, Sh.JS performed administrative, technical, and material support. All authors read and approved the final manuscript.

\section{Acknowledgments}

Department of Midwifery and Reproductive Health of Tarbiat Modares University Medical School supported this study. Therefore, we would like to thank the staff of Taleghani Educational Hospital and all the women who participated in the present study.

\section{References}

1. Jacobs RJ, Collias BA, Rana AM, Wallace EM, Kane MN, Boesler DR. Identification of musculoskeletal disorders in medically underserved regions of South America and Vietnam. J Am Osteopath Assoc. 2015;115:12-22.

2. Leveille SG, Zhang Y, McMullen W, Kelly-Hayes M, Felson DT. Sex differences in musculoskeletal pain in older adults. Pain. 2005;116:332-8.

3. Berkley KJ. Sex differences in pain. Behav Brain Sci. 1997;20:371-80. discussion 435-513.

4. Ahn S, Song R. Bone mineral density and perceived menopausal symptoms: factors influencing low back pain in postmenopausal women. J Adv Nurs. 2009;65:1228-36.

5. Blumel JE, Chedraui P, Baron G, Belzares E, Bencosme A, Calle A, et al. Menopause could be involved in the pathogenesis of muscle and joint aches in mid-aged women. Maturitas. 2013;75:94-100.

6. Berek JS. Berek \& Novak's Gynecology. 15 ed: Golban Nashr Company; 2012. 1384 p.

7. Blumel JE, Palacios S, Legorreta D, Vallejo MS, Sarra S. Is fibromyalgia part of the climacteric syndrome? Maturitas. 2012;73:87-93.

8. Kahn MF. Does hormone replacement therapy discontinuation cause musculoskeletal pain? Joint Bone Spine. 2006;73:488-9.

9. Braden JB, Young A, Sullivan MD, Walitt B, Lacroix AZ, Martin L. Predictors of change in pain and physical functioning among post-menopausal women with recurrent pain conditions in the women's health initiative observational cohort. J Pain. 2012;13:64-72.

10. Yip YB, Ho SC, Chan SG. Socio-psychological stressors as risk factors for low back pain in Chinese middle-aged women. J Adv Nurs. 2001;36:409-16.

11. de Kruijf M, Stolk L, Zillikens MC, de Rijke YB, Bierma-Zeinstra SM, Hofman A, et al. Lower sex hormone levels are associated with more chronic musculoskeletal pain in community-dwelling elderly women. Pain. 2016;157:1425-31.

12. Mitchell ES, Woods NF. Pain symptoms during the menopausal transition and early postmenopause. Climacteric. 2010;13:467-78.

13. Kim HM, Park J, Ryu SY, Kim J. The effect of menopause on the metabolic syndrome among Korean women: the Korean National Health and Nutrition Examination Survey, 2001. Diabetes Care. 
2007;30:701-6.

14. Heinemann K, Ruebig A, Potthoff P, Schneider HP, Strelow F, Heinemann LA, et al. The Menopause Rating Scale (MRS) scale: a methodological review. Health Qual Life Outcomes. 2004;2:45.

15. Cuesta-Vargas Al, Gonzalez-Sanchez M. Spanish version of the screening Orebro musculoskeletal pain questionnaire: a cross-cultural adaptation and validation. Health Qual Life Outcomes. 2014;12:157.

16. Dagfinrud H, Storheim K, Magnussen LH, Odegaard T, Hoftaniska I, Larsen LG, et al. The predictive validity of the Orebro Musculoskeletal Pain Questionnaire and the clinicians' prognostic assessment following manual therapy treatment of patients with LBP and neck pain. Man Ther. 2013;18:124-9.

17. Akkus S, Delibas N, Tamer MN. Do sex hormones play a role in fibromyalgia? Rheumatology. 2000;39:1161-3.

18. Sambrook PN, Eisman JA, Champion GD, Pocock NA. Sex hormone status and osteoporosis in postmenopausal women with rheumatoid arthritis. Arthritis Rheum. 1988;31:973-8.

19. Nikolova V, Petkova M. Pain sensitivity among women with low estrogen levels. Procedia - Social Behavioral Sciences. 2010;5:289-93.

20. Sowers MR, McConnell D, Jannausch M, Buyuktur AG, Hochberg M, Jamadar DA. Estradiol and its metabolites and their association with knee osteoarthritis. Arthritis Rheum. 2006;54:2481-7.

21. Ogwumike O, Adeniyi A, Orogbemi O. Musculoskeletal pain among postmenopausal women in Nigeria: Association with overall and central obesity. Hong Kong Physiotherapy Journal 26 july 2015.

22. Sipila S. Body composition and muscle performance during menopause and hormone replacement therapy. J Endocrinol Invest. 2003;26:893-901.

23. Roman-Blas JA, Castaneda S, Largo R, Herrero-Beaumont G. Osteoarthritis associated with estrogen deficiency. Arthritis Res Ther. 2009;11:241.

24. Stening KD, Eriksson O, Henriksson KG, Brynhildsen J, Lindh-Astrand L, Berg G, et al. Hormonal replacement therapy does not affect self-estimated pain or experimental pain responses in postmenopausal women suffering from fibromyalgia: a double-blind, randomized, placebo-controlled trial. Rheumatology. 2011;50:544-51.

25. Magliano M. Menopausal arthralgia: Fact or fiction. Maturitas. 2010;67:29-33.

26. Barnabei VM, Cochrane BB, Aragaki AK, Nygaard I, Williams RS, McGovern PG, et al. Menopausal symptoms and treatment-related effects of estrogen and progestin in the Women's Health Initiative. Obstet Gynecol. 2005;105:1063-73.

27. Wijnhoven HA, de Vet HC, Smit HA, Picavet HS. Hormonal and reproductive factors are associated with chronic low back pain and chronic upper extremity pain in women-the MORGEN study. Spine (Phila Pa 1976). 2006;31:1496-502.

28. Sievert LL, Goode-Null SK. Musculoskeletal pain among women of menopausal age in Puebla, Mexico. J Cross Cult Gerontol. 2005;20:127-40. 
29. Gao HL, Lin SQ, Wei Y, Chen Y, Wu ZL. The effect of age and menopausal status on musculoskeletal symptoms in Chinese women aged 35-64 years. Climacteric. 2013;16:639-45.

30. Dugan SA. Exercise for health and wellness at midlife and beyond: balancing benefits and risks. Phys Med Rehabil Clin N Am. 2007;18:555-75, xi. 